\title{
Variabilité des caractéristiques des fromages saint-nectaire fermiers : relations avec la composition du lait et les conditions de production
}

\author{
Claire Agabriel $^{\mathrm{a} *}$, Jean-Baptiste Coulon ${ }^{\mathrm{b}}$, Chantal Journal ${ }^{\mathrm{a}}$, \\ Cécile Sibra ${ }^{a}$, Hélène Albouyc
}

a Département agriculture et espace, ENITA, 63370 Lempdes, France

${ }^{\mathrm{b}}$ Laboratoire adaptation des herbivores aux milieux, Inra,

63122 Saint-Genès Champanelle, France

${ }^{\mathrm{c}}$ ENILV, BP 537, 15005 Aurillac cedex, France

Reçu le 15 mai 1998 ; accepté le 22 décembre 1998

\begin{abstract}
Effect of milk composition and herd management conditions on the characteristics of farmhouse saint-nectaire cheese. This study was performed in 23 farms producing raw milk saint-nectaire cheeses, chosen on their good processing ability. Chemical and sensory analysis were done on 67 cheeses made in these farms during winter. Important variability of chemical and sensory characteristics was observed between cheeses. Six main groups of cheeses were identified, based on sensory characteristics, texture and flavour descriptors being the most discriminant. Chemical and bacteriological characteristics of milk were not very different between groups. Differences between groups were associated either to processing parameters (type of starters) or feeding conditions of the cows (concentrate proportion in the diet). The type of roughage (hay vs fermented roughage) was not different between groups of cheeses. (C) Inra/Elsevier, Paris.
\end{abstract}

\section{cheese / feeding / sensory characteristic}

Résumé - Soixante-sept fromages ont été collectés au cours de la période hivernale chez 23 producteurs de saint-nectaire fermier, choisis pour leur maîtrise de la technologie de fabrication. Les caractéristiques physico-chimiques et sensorielles ont été très variables d'un fromage à l'autre. Six grandes classes de fromages ont été identifiées sur la base des caractéristiques sensorielles. Elles opposent les fromages sur deux grands groupes de critères liés à la texture et à la flaveur. Les caractéristiques chimiques et bactériologiques des laits mis en œuvre n'ont pas été très différentes d'une classe à l'autre. Ces différentes classes sont associées à des pratiques de transformation différentes (type de ferments). La nature de la ration de base offerte aux vaches n'a pas été significativement différente d'une classe à l'autre. Par contre, la proportion de concentré dans la ration, qui est un indicateur

* Correspondance et tirés à part. agabriel@gentiane.enitac,fr 
indirect de l'intensification de la conduite du troupeau, a été plus faible dans les classes 1 et 2 qui regroupent les fromages les plus typés. La nature du travail effectué ne permet cependant pas d'affirmer qu'il s'agit là d'une relation de cause à effet. (O) Inra/Elsevier, Paris.

\section{fromage / alimentation / caractéristique sensorielle}

\section{INTRODUCTION}

Les caractéristiques des fromages affinés dépendent à la fois de la technologie de fabrication et de la composition physicochimique et bactériologique des laits mis en oeuvre. Cette dernière est liée en partie aux conditions de production, et en particulier à l'alimentation des animaux. Dans le cas de certains fromages d'appellation d'origine contrôlée, où les modifications du lait sont limitées, voire interdites, ces conditions de production pourraient avoir des effets sensibles sur les caractéristiques physico-chimiques et sensorielles des fromages affinés. Lorsque les fromages sont fabriqués à la ferme, à partir du lait d'un seul troupeau, les variations de la composition du lait au cours de l'année, parfois très importantes, pourraient se répercuter directement sur les propriétés des fromages. Les productions fermières représentent ainsi un modèle particulièrement intéressant pour étudier ces relations. Cependant, s'il est admis, à partir d'observations empiriques, que l'alimentation des animaux influe sur la qualité des fromages [17], il existe encore peu de travaux sur ce thème réalisés sur des fromages fermiers $[11,14]$.

L'objectif de cette étude est d'une part, de mettre en évidence la variabilité des caractéristiques d'un fromage fermier (saint-nectaire fermier) et d'autre part, d'analyser cette variabilité en fonction des conditions de production du lait. Le choix des producteurs a été réalisé de manière à minimiser les effets de la technologie (conditions de fabrication et d'affinage du fromage) afin de s'attacher plus spécifiquement aux facteurs d'amont, et en particulier à l'alimentation des animaux.

\section{MATÉRIEL ET MÉTHODES}

\subsection{Données}

Vingt trois producteurs de saint-nectaire fermier, commercialisant leurs fromages en blanc auprès de trois affineurs différents ont été retenus dans cette étude. Ces producteurs avaient été proposés par les affineurs en raison de leur maîtrise de la technologie de fabrication et de la régularité de qualité des fromages livrés. Ces exploitations ont fait l'objet d'une enquête au cours de l'hiver 1996 , portant plus particulièrement sur les pratiques d'alimentation des animaux (nature des fourrages et des concentrés offerts au cours de l'hiver), la conduite du troupeau (logement et propreté des vaches laitières, pratiques de traite, répartition des vêlages) et les conditions de fabrication des fromages.

Sur chacune de ces exploitations, trois fois au cours de l'hiver, un lot de fromages issus de la transformation de la totalité du lait de la traite du matin a été identifié et affiné par l'affineur habituel pendant une même durée de sept semaines. Le pH (à $20^{\circ} \mathrm{C}$ ), l'extrait sec (par dessiccation à $102{ }^{\circ} \mathrm{C}$ pendant $24 \mathrm{~h}$ ) et la teneur en matières grasses (par butyrométrie [9]) ont été mesurés sur un fromage représentatif de chaque lot. Ce même fromage a ensuite été soumis à un jury d'analyse sensorielle, composé de dix sujets bénévoles, entraînés à la reconnaissance des saveurs élémentaires [1]. Ce jury a préalablement été exercé à l'évaluation de diverses caractéristiques (aspect, odeur, goût, texture) de fromages de saint-nectaire. Un profil du produit a ensuite été établi par génération libre de descripteurs dont le nombre a été réduit avec l'accord du panel de dégustateurs $[2,5]$. Les sujets ont été entraînés à l'évaluation de l'intensité de chacun des descripteurs du profil définitif. La notation a été effectuée sur une échelle structurée de 0 à 10. Le profil comportait deux parties d'une quinzaine de descripteurs chacun, l'une concernant la description du produit en bouche, et l'autre l'aspect du fromage. Les sujets ont toujours commencé par la partie dégustation pro- 
prement dite : les échantillons de fromage écroûtés étaient codés et présentés simultanément à tous les dégustateurs dans un ordre aléatoire et différent pour chacun. La partie « aspect »du fromage a été évaluée sur un quart du fromage, codé de manière différente de la partie précédente.

À chaque séance, cinq fromages, provenant d'au moins deux affineurs différents, ont été dégustés. Au total, compte tenu que deux fromages n'ont pas pu être récupérés auprès des affineurs, 67 fromages, âgés de 50 à $59 \mathrm{j}$, ont été dégustés au cours de 13 séances réparties sur quatre mois.

Chacun des trois matins de fabrication, un échantillon de lait de cuve a été prélevé pour mesure du $\mathrm{pH}\left(\mathrm{a} 20^{\circ} \mathrm{C}\right)$, détermination du taux d'urée (méthode colorimétrique au diméthyl amino benzaldéhyde), de la teneur en protéines coagulables (différence entre taux protéique du Iait et taux protéique du sérum après coagulation à la présure), et du degré de lipolyse du lait [9]. Par ailleurs, les caractéristiques du lait ont été complétées à partir des analyses mensuelles du lait de cuve réalisées par le syndicat du saintnectaire. Les mesures des taux butyreux et protéique, de la numération cellulaire (Somacount), des teneurs en spores butyriques [6], en bactéries coliformes [3] et en Staphylococcus aureus [4] utilisées correspondaient aux résultats de l'analyse mensuelle effectuée à la date la plus proche du jour de fabrication des fromages étudiés (écart moyen de $8 \mathrm{j}$ entre la date du prélèvement de lait et celle de fabrication du fromage).

Les éleveurs ont noté les caractéristiques de l'alimentation distribuée aux vaches laitières pendant la semaine de chaque fabrication identifiée. Pendant l'hiver, les fourrages fermentés utilisés (ensilage d'herbe et enrubannage) ont fait l'objet d'un prélèvement sur lequel le $\mathrm{pH}$, la teneur en matière sèche, en azote ammoniacal et en spores butyriques ont été déterminés.

Enfin, les conditions de fabrication ont été relevées par le producteur, au cours de chacune des trois fabrications hivernales. Ces conditions concernaient essentiellement le matériel utilisé, la maturation du lait (nature des ferments, température, durée), sa coagulation (température, dose de présure, temps de coagulation total), les températures de la tomme et des locaux lors du découpage du caillé et du moulage, les durées de brassage du mélange caillé-lactosérum, du moulage, du pressage, ainsi que la durée totale de fabrication. La température du local de préaffinage a également été relevée.

\subsection{Analyse des données}

La démarche générale a été de constituer des classes de fromages présentant des caractéristiques sensorielles homogènes, puis d'analyser les différences entre ces classes en fonction, d'une part des caractéristiques physico-chimiques des fromages, d'autre part des conditions de fabrication et d'affinage, enfin des caractéristiques du lait, des animaux (race, stade physiologique) et de leur conduite (essentiellement alimentaire).

Les classes de fromages ont été élaborées à l'aide d'une classification ascendante hiérarchique $(\mathrm{CAH}$, logiciel SPAD) construite à partir des résultats d'une analyse en composantes principales (ACP, coordonnées des fromages sur les cinq premiers axes de l'analyse). Cette ACP a été réalisée à partir des caractéristiques sensorielles concernant la pâte de 64 fromages. Dixhuit descripteurs ont été retenus, trois concernant l'aspect, six la texture et neuf la flaveur de la pâte. Trois fromages, provenant du même producteur, sont en effet apparus, dans un premier traitement des données, très différents des autres sur tous les critères sensoriels et physico-chimiques. Ces fromages n'ont pas été introduits dans l'analyse définitive, de manière à ne pas la biaiser. Ils constituent à eux seuls une classe de fromages (classe S) qui sera décrite dans les résultats. Les variables explicatives (conduite des animaux, caractéristiques du lait, conditions de fabrication fromagère) ont été traitées par analyse de variance à un facteur (la classe de fromage) lorsqu'elles étaient quantitatives, ou par le test du chi deux lorsqu'elles étaient qualitatives [I6].

\section{RÉSULTATS}

\subsection{Caractéristiques des exploitations}

La surface agricole utile des 23 exploitations variait de 38 à 181 ha, et leur altitude de 780 à $1250 \mathrm{~m}$. Les troupeaux étaient composés de 25 à 100 vaches, la plupart de race montbéliarde (15 troupeaux sur 23), logés essentiellement en stabulation entravée (18 fermes sur 23). L'état de propreté des animaux, noté selon la méthode proposée par Faye et Barnouin [8] était médiocre (note moyenne $6,1 / 10$ : plus la note est élevée, moins les animaux sont propres) bien que des différences importantes entre troupeaux 
aient été relevées (notes de 3,5 à 8). De même, l'hygiène au cours de la traite était très variable : notes étalées de 1 à 9 sur 10 (note élaborée à partir des pratiques de nettoyage de la mamelle avant et après la traite, de l'élimination des premiers jets et de l'ordre de traite des animaux malades).

En hiver, les animaux étaient nourris soit avec des rations exclusivement à base de foin (cinq exploitations), soit avec du foin complété par de l'ensilage d'herbe (six exploitations) ou de l'enrubannage (dix exploitations). Trois éleveurs utilisaient exclusivement des fourrages fermentés pendant tout ou partie de 1'hiver. Dans six exploitations, les animaux étaient encore au pâturage lors de la fabrication des premiers lots de fromages.

\subsection{Conditions de fabrication des fromages}

Tous les producteurs fabriquaient le fromage deux fois par jour à partir du lait entier cru d'une seule traite. Cette fabrication avait lieu dans un local spécifique dont les caractéristiques sont variables. Dans la majorité des cas, le matériel utilisé (cuve, outils de décaillage et de brassage, presse, étagères de stockage) était en acier inoxydable et en plastique. Seuls cinq producteurs utilisaient encore du bois (quatre rassemblent la tomme avec une planche en bois, un dispose les fromages sur des étagères en bois).

Dans 22 fermes sur 23, le lait entier cru était additionné de levain (ferments thermophiles seuls pour 11 producteurs, associés à des mésophiles dans 11 exploitations). La maturation a duré en moyenne $69 \mathrm{~min}(0)$ à $155 \mathrm{~min}$ ). L'emprésurage, avec une dose de présure à $520 \mathrm{~mL}$ de chymosine active par litre allant de 15 à $40 \mathrm{~mL} \cdot 100 \mathrm{~L}^{-1}$, était réalisé à une température moyenne de $33{ }^{\circ} \mathrm{C}$ (de 31 à $34,3^{\circ} \mathrm{C}$ ). Le temps total de coagulation a varié de 30 à $75 \mathrm{~min}$ (moyenne $45 \mathrm{~min}$ ). Dans 11 fabrications, de l'eau chaude a été ajoutée avant le décaillage pour réchauffer la tomme. Le décaillage était réalisé manuel- lement dans la plupart des exploitations (trois producteurs disposaient d'une cuve mécanisée). Dans la fabrication de 54 fromages le mélange caillé-lactosérum était brassé (durée moyenne $9 \mathrm{~min}$ allant de 2 à 20 min). Le sérum était éliminé au seau ou par gravité par 14 producteurs, les neuf autres utilisaient une pompe et quatre pratiquaient le délactosage (fabrication de 12 fromages). Le travail en cuve (emprésurage jusqu'au début du moulage) a duré en moyenne $75 \mathrm{~min}$ (50 à $135 \mathrm{~min}$ ). Le caillé était moulé presque toujours avec une prépresse automatique ( 22 fermes sur 23 ). Les fromages étaient salés à sec au cours de leur retournement pendant le moulage. Au début du moulage, la température de la tomme était en moyenne de $32,5^{\circ} \mathrm{C}$. Elle restait en général stable pendant la durée du moulage (chute d'environ $1^{\circ} \mathrm{C}$ ). La durée de la fabrication (mise des ferments jusqu'à la fin du moulage) était très variable (de 75 à $300 \mathrm{~min}$ ). Les fromages étaient en général pressés dans le local de fabrication pendant 12 ou $24 \mathrm{~h}$. Ils étaient stockés dans un local de préaffinage (température moyenne $8,5^{\circ} \mathrm{C}$ ) jusqu'à leur départ chez un affineur, deux à six jours après leur fabrication.

\subsection{Caractéristiques du lait}

La composition chimique du lait a été très variable d'une fabrication à l'autre. Le taux butyreux a varié de 26,4 à $51,5 \mathrm{~g} \cdot \mathrm{L}^{-1}$ (moyenne $38,9 \mathrm{~g} \cdot \mathrm{L}^{-1}$ ), le taux protéique de 28,8 à $36,7 \mathrm{~g} \cdot \mathrm{L}^{-1}$ (moyenne $33,0 \mathrm{~g} \cdot \mathrm{L}^{-1}$ ) et le taux d'urée de 0,15 à $0,45 \mathrm{~g} \cdot \mathrm{L}^{-1}$ (moyenne $\left.0,28 \mathrm{~g} \cdot \mathrm{L}^{-1}\right)$. Le $\mathrm{pH}$ du lait a varié de 6,45 à 6,74 (moyenne 6,66), mais la grande majorité des valeurs $(85 \%)$ a été comprise entre 6,61 et 6,69 .

La lipolyse du lait était généralement limitée puisque les trois quarts des laits ont présenté une valeur inférieure à $0,12 \mathrm{~g} \mathrm{C}_{18} \cdot 100 \mathrm{~g}^{-1}$. La qualité hygiénique des laits était variable d'un prélèvement à l'autre. Les deux tiers des prélèvements ont présenté une numération cellulaire inférieure 
à 300000 cellules $\cdot \mathrm{mL}^{-1}$, et $70 \%$ ont présenté moins de 100 coliformes $\cdot \mathrm{mL}^{-1}$. La contamination butyrique était inférieure à 500 spores $\mathrm{L}^{-1}$ dans $60 \%$ des prélèvements et celle en Staphylococcus aureus a été inférieure à $100 \cdot \mathrm{mL}^{-1}$ dans $48 \%$ d'entre eux.

\subsection{Caractéristiques des fromages}

\subsubsection{Moyennes et variabilité}

Les analyses chimiques et sensorielles ont été réalisées sur des fromages âgés de $56 \mathrm{j}$ en moyenne. Une variabilité importante a été relevée aussi bien pour leurs caractéristiques chimiques que sensorielles (tableau I). L'extrait sec total, le rapport gras/sec et le
$\mathrm{pH}$ des fromages affinés ont varié respectivement entre 47 et $59,2 \%$, entre 48,3 et $59,8 \%$ et entre 5,2 et 6,2 . Les notes des descripteurs visuels ont présenté des moyennes assez homogènes (moyenne 4,3 allant de 3,4 pour le caractère poisseux à 5 pour la fermeté) et une variabilité importante (étendue moyenne de 6,7 allant de 4,4 pour l'élasticité à 8,7 pour la présence de mucor). Les notes des descripteurs en bouche ont été plus faibles (moyenne 3,8 allant d'une moyenne de 1,3 pour le goût rance à 5,5 pour l'intensité du goût) et leur variabilité moins grande (étendue moyenne de 4,1 allant de 2,2 pour le caractère agréable de l'odeur à 7,3 pour la fermeté de la texture de la pâte).

Tableau I. Composition chimique et caractéristiques sensorielles des fromages affinés $(n=67)$.

Table I. Chemical composition and sensory characteristics of ripened cheeses $(n=67)$.

\begin{tabular}{lcccc}
\hline & moyenne & minimum & maximum & écart type \\
\hline Âge (j) & 48,4 & 40 & 57 & 3,0 \\
pH & 5,61 & 5,15 & 6,15 & 0,23 \\
Matière sèche (\%) & 53,0 & 47,0 & 59,2 & 2,7 \\
Matières grasses (\%) & 28,0 & 23,5 & 33,0 & 2,2 \\
Extrait sec dégraissé (\%) & 25,0 & 22,0 & 29,7 & 1,6 \\
& & & & \\
Caractéristiques sensorielles (/10) & & 1,0 & 7,2 & 1,6 \\
aspect déformé & 4,0 & 1,9 & 7,1 & 1,2 \\
intensité de la couleur de la pâte & 4,1 & 0,1 & 8,6 & 2,1 \\
quantité d'ouvertures & 3,9 & 0,2 & 8,1 & 1,8 \\
taille des ouvertures & 4,4 & 1,1 & 6,6 & 1,2 \\
texture collante & 4,2 & 0,9 & 7,7 & 1,5 \\
texture fondante & 4,2 & 0,8 & 8,1 & 1,8 \\
texture ferme & 4,5 & 0,6 & 5,4 & 0,9 \\
texture granuleuse & 2,1 & 4,0 & 7,2 & 0,7 \\
intensité du goût & 5,5 & 2,9 & 6,8 & 0,8 \\
persistance du goût & 5,2 & 2,6 & 5,8 & 0,7 \\
goût typique & 4,5 & 0,2 & 3,1 & 0,8 \\
goût rance & 1,3 & 0,8 & 4,4 & 0,6 \\
goût acide & 2,2 & 0,4 & 3,8 & 0,8 \\
goût amer & 1,7 & 2,7 & 5,7 & 0,7 \\
goût salé & 4,2 & 3,8 & 6,8 & 0,7 \\
intensité de l'odeur & 5,2 & 4,0 & 6,2 & 0,5 \\
odeur agréable & 5,0 & 2,1 & 6,4 & 1,0 \\
satisfaction globale en bouche & 4,5 & & & \\
\hline
\end{tabular}


Certains descripteurs sensoriels ont été fortement liés entre eux ou aux caractéristiques chimiques du fromage. C'est le cas des descripteurs texture fondante et texture collante $(R=+0,61)$, texture ferme et texture granuleuse $(R=+0,66)$, et surtout texture ferme et texture fondante $(\mathrm{R}=-0,91)$. L'intensité et la persistance du goût étaient aussi fortement liés $(\mathrm{R}=+0,79)$, de même que les teneurs en matière sèche et en matières grasses $(\mathrm{R}=+0,84)$.

\subsubsection{Typologie des fromages selon leurs caractéristiques}

L'ACP réalisée sur les 64 fromages permet de dégager deux grands axes de variation (figure 1). Le premier oppose des fromages fermes et granuleux à des fromages présentant une texture collante et fondante, un goût intense et persistant et une odeur intense. Le second est essentiellement un axe de flaveur ; il oppose des fromages présentant un goût typique, salé, avec une odeur agréable à des fromages présentant un goût amer et rance. À partir de cette ACP, en plus des trois fromages très particuliers du même producteur (classe S), six classes de fromages ont été constituées (figure 2).

La classe I regroupe sept fromages ayant la texture de pâte la plus fondante, la plus collante, et la moins granuleuse. Peu fermes, ils se déforment et sont « détalonnés », ce qui peut expliquer leur mauvaise appréciation visuelle. Leur pâte présente assez peu d'ouvertures. Leur odeur est agréable et la plus intense. Leur goût est aussi le plus intense et le plus persistant, le plus typique, le plus salé, le plus acide, peu amer et peu rance. Ces fromages sont pauvres en matière sèche et notamment en matières grasses ; leur extrait sec dégraissé est très faible. Ils proviennent de cinq producteurs différents (dont un qui fournit trois fromages). Ils ont été affinés chez deux affineurs.

Le goût et l'odeur des 13 fromages de la classe II sont proches de ceux de la classe I, mais moins intenses et moins persistants. Leur texture est la plus ferme ; ce sont donc

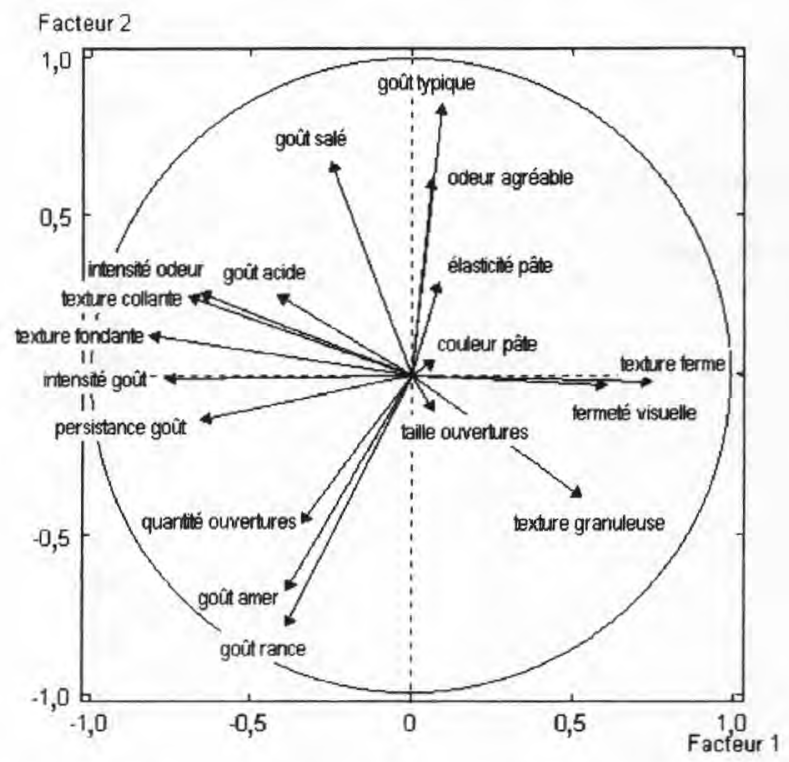

Figure 1. Cercle des corrélations des 18 variables actives de l'analyse en composantes principales.

Figure 1. Correlation circle of 18 active variables of analysis in main components. 


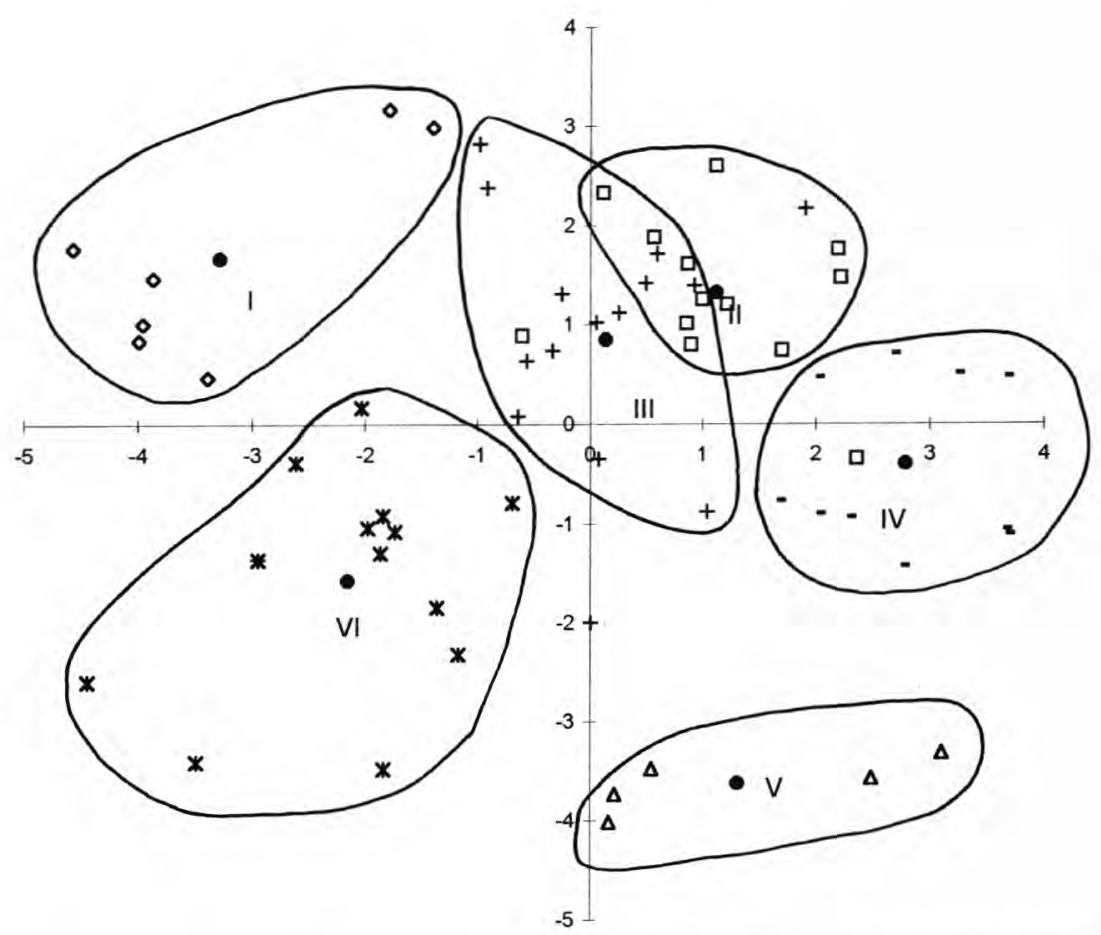

• Classe I ם Classe II + Classe III - Classe IV $\Delta$ Classe V $*$ Classe VI $\bullet$ Centres de gravité

Figure 2. Représentation des fromages sur le plan 1-2.

Figure 2. Graphic representation of cheeses on map 1-2.

les fromages les moins déformés dont la surface est la plus régulière. Leur pâte présente moins d'ouvertures. Leur teneur en matières grasses et leur extrait sec dégraissé sont élevés. Ce sont les fromages qui ont été le plus apprécié, aussi bien visuellement qu'en bouche. Ils ont été fabriqués par huit producteurs et affinés dans deux caves.

La classe III est numériquement la plus importante. Elle est constituée de 16 fromages (dix producteurs différents, affinés par trois affineurs) dont les caractéristiques physico-chimiques, de texture et d'odeur sont moyennes, avec un goût typique, très peu acide et très peu rance. Leur pâte présente peu d'ouvertures, mais larges.

La classe IV regroupe dix fromages dont l'odeur et le goût sont les moins intenses et les moins persistants. Tous les descripteurs de goût sont faibles. Leur texture est ferme. Comme pour les fromages de la classe III, leurs caractéristiques physico-chimiques sont moyennes et leur pâte présente peu d'ouvertures, mais larges. Ils proviennent de sept producteurs différents et ont été affinés par les trois affineurs.

Les caractéristiques des cinq fromages (trois producteurs, deux affineurs) de la classe $\mathrm{V}$ sont proches de celles des fromages de la classe IV, mais avec une texture plus granuleuse et moins élastique, une teneur en matière sèche plus élevée. Ce sont les fromages les plus amers et les plus rances.

La classe VI regroupe 13 fromages (neuf producteurs, trois affineurs) dont le goût est intense et persistant, avec une acidité, une 
amertume et une saveur rance accentuées. Les caractéristiques de texture sont moyennes. La pâte présente les ouvertures les plus nombreuses et les plus grandes. Ce sont, avec les fromages de la classe $\mathrm{V}$, les fromages les moins appréciés, aussi bien visuellement qu'en bouche. Comme les fromages de la classe I, leurs teneurs en matières sèche et grasse et leurs extraits secs dégraissés sont faibles.

Les caractéristiques des trois fromages $S$ présentent les mêmes tendances que celles de la classe V mais plus accentuées. Ce sont les fromages les plus fermes et les plus granuleux et dont les teneurs en matière sèche et extrait sec dégraissé sont les plus fortes. Ils ont très peu d'odeur et de goût, mis à part un goût rance le plus prononcé. Le lait utilisé a été le plus pauvre en protéines coagulables ; il a présenté le $\mathrm{pH}$ et le niveau de lipolyse les plus élevés.

On a utilisé pour leur fabrication des ferments thermophiles avec une dose de présure faible. Malgré une courte durée de fabrication, la température n'était pas très stable.

Si la stabulation a été notée la plus propre, l'hygiène de la traite a été la plus défavorable.

\subsection{Liaisons avec les caractéristiques du lait, la transformation et les pratiques de conduites des vaches}

Les caractéristiques du lait ont été relativement voisines d'une classe de fromages à l'autre (tableau II). Seule la classe VI se distingue nettement des autres, avec un lait plus riche en matières utiles et en urée et un pH élevé, ce qui peut expliquer en partie les particularités observées sur les fromages de cette classe [15]. Les laits de la classe I ont présenté une numération cellulaire supérieure à celle des autres classes $(p<0,05)$, et ceux de la classe II une contamination en coliformes supérieure à celle des autres classes. Il n'y a pas eu de différence majeure entre les classes de fromages pour la contamination en spores butyriques et en Staphylococcus aureus.

Les conditions de fabrication du fromage ont souvent été aussi variables à l'intérieur des classes de fromage qu'entre classes (tableau II). Cependant, malgré le caractère essentiellement qualitatif de l'appréciation de ces conditions de fabrication, nous avons pu observer que les fromages des classes I et II étaient associés à des fabrications utilisant peu de présure et un mélange de ferments mésophiles et thermophiles, alors que ceux des classes III et surtout IV étaient associés à des fabrications utilisant beaucoup de présure et des ferments uniquement thermophiles. Par contre, les fromages des classes V et VI ont été fabriqués avec une très grande variabilité de pratiques. Par ailleurs, les classes I et V s'opposent nettement par l'évolution de la température entre l'emprésurage et le milieu du moulage, ainsi que par la durée de fabrication (de l'addition des ferments jusqu'à la fin du moulage).

En ce qui concerne l'alimentation des animaux, on observe une grande variabilité du type de ration à l'intérieur de chaque classe de fromages, même si les rations à base de foin semblent dominantes dans les classes I, IV et V (tableau III). Les caractéristiques des ensilages et des balles rondes enrubannées (teneur en matière sèche, $\mathrm{pH}$ et teneur en azote ammoniacal) n'étaient pas différentes d'une classe à l'autre. En revanche, la part de concentré dans la ration était significativement plus importante dans les classes III, IV et V que dans les classes I, II et VI. La propreté de la stabulation était la moins bonne dans les classes I et VI, et l'hygiène de la traite la moins maîtrisée dans les classes I et V, mais ces écarts n'ont pas été significatifs.

\section{DISCUSSION ET CONCLUSION}

Cette étude a été réalisée sur un échantillon particulier de producteurs choisis pour 
Tableau II. Relation entre les classes de fromages et les caractéristiques du lait, les conditions de fabrication.

Table II. Relation between groups of cheeses and characteristics of milk, cheesemaking conditions.

\begin{tabular}{lllllllll}
\hline Classes de fromages & I & II & III & IV & V & VI & S & Sign $^{1}$ \\
\hline nombre de fromages & 7 & 13 & 16 & 10 & 5 & 13 & 3 & \\
\hline
\end{tabular}

\section{Caractéristiques du lait}

\begin{tabular}{|c|c|c|c|c|c|c|c|c|}
\hline $\mathrm{pH}$ & 6,65 & 6,63 & 6,66 & 6,65 & 6,64 & 6,68 & 6,7 & + \\
\hline teneur en protéines coagulables $\left(\mathrm{g} \cdot \mathrm{L}^{-1}\right)$ & 27,4 & 27,5 & 27,2 & 26,9 & 27,4 & 28,4 & 24,9 & + \\
\hline taux d'urée $\left(\mathrm{mg} \cdot 100 \mathrm{~mL}^{-1}\right)$ & 0,28 & 0,26 & 0,29 & 0,24 & 0,25 & 0,32 & 0,24 & + \\
\hline lipolyse $\left(\mathrm{g} \mathrm{C}_{18} .100 \mathrm{~g}^{-1}\right)$ & 0,12 & 0,10 & 0,13 & 0,11 & 0,08 & 0,17 & 0,20 & NS \\
\hline taux protéique $\left(\mathrm{g} \cdot \mathrm{L}^{-1}\right)$ & 33,2 & 33,1 & 32,7 & 32,3 & 33,0 & 34,4 & 29,6 & $*$ \\
\hline taux butyreux $\left(\mathrm{g} \cdot \mathrm{L}^{-1}\right)$ & 38,0 & 39,2 & 38,8 & 38,2 & 38,0 & 40,1 & 38,2 & NS \\
\hline cellules $\left(\log \cdot m L^{-1}\right)$ & 5,8 & 5,5 & 5,3 & 5,3 & 5,4 & 5,3 & 5,4 & \\
\hline liformes $\left(\log \cdot m L^{-1}\right)$ & 1,1 & 2,1 & 1,5 & 1,3 & 1,8 & 1,2 & 0,7 & * \\
\hline aphylocoques $\left(\log \cdot m L^{-1}\right)$ & 2,2 & 2,2 & 1,9 & 1,8 & 2,2 & 1,6 & 1,3 & NS \\
\hline ores butyriques $\left(\log \cdot \mathrm{mL}^{-1}\right)$ & 2,3 & 3,0 & 2,6 & 2,5 & 2,9 & 2,5 & 2,6 & NS \\
\hline
\end{tabular}

\section{Conditions de fabrication}

type de ferments (nombre d'ateliers)

- aucun ferment

- thermophiles

- thermophiles et mésophiles

dose de présure ( $\mathrm{mL} \cdot 100 \mathrm{~L}^{-1}$ de lait)

chute de température entre l'empré-

surage et le milieu du moulage $\left({ }^{\circ} \mathrm{C}\right)$

temps écoulé entre la mise des ferments

et la fin du moulage ( $\mathrm{min}$ )

durée d'affinage (j)

$\begin{array}{rrrrrrr}1 & 0 & 0 & 0 & 1 & 3 & 0 \\ 0 & 3 & 10 & 9 & 2 & 4 & 3 \\ 6 & 10 & 6 & 1 & 2 & 6 & 0 \\ 24 & 26 & 31 & 31 & 28 & 28 & 23\end{array}$

$\begin{array}{llllllll}2,4 & 1,1 & 1,0 & 1,1 & 0,7 & 1,8 & 1,8 & * *\end{array}$

$\begin{array}{rrrrrrrr}167 & 194 & 179 & 194 & 240 & 208 & 163 & + \\ 47 & 49 & 49 & 48 & 49 & 47 & 51 & \text { NS }\end{array}$

Conditions d'affinage (nombre d'ateliers)

Affineur 1

Affineur 2

Affineur 3

$\begin{array}{rrrrrrr}4 & 3 & 5 & 4 & 2 & 7 & 0 \\ 0 & 0 & 6 & 4 & 0 & 5 & 3 \\ 3 & 10 & 5 & 2 & 3 & 1 & 0\end{array}$

\footnotetext{
${ }^{1}$ signification statistique : NS : non significatif, $+: 10 \%, *: 5 \%, * *: 1 \%$. L’analyse statistique a été réalisée sur les 64 fromages des classes 1 à 6 .

'statistical signification: NS: non significant, $+: 10 \%, *: 5 \%, * *: 1 \%$. The statistical analysis was realized on 64 cheeses from the groups 1 to 6 .
}

leur régularité en matière de qualité des fromages. En effet, si six exploitations seulement sur 23 ont leurs trois fromages dans la même classe, seules trois exploitations ont présenté leurs trois fromages dans trois classes différentes. Il existe donc bien, dans notre échantillon, une certaine stabilité dans la qualité des fromages de chaque produc- teur. La variabilité des caractéristiques physico-chimiques et sensorielles a cependant été importante, en particulier en ce qui concerne la texture, d'autant plus qu'aucun fromage fabriqué au printemps ou en été n'a été étudié, périodes pendant lesquelles sont souvent observées des variations importantes de qualité des fromages [10,13]. Cette 
Tableau III. Relation entre les classes de fromages et la conduite des animaux.

Table III. Relation between groups of cheeses and animal management.

\begin{tabular}{lllllllll}
\hline Classes de fromages & I & II & III & IV & V & VI & S & Sign \\
\hline nombre de fromages & 7 & 13 & 16 & 10 & 5 & 13 & 3 & \\
\hline
\end{tabular}

Type de vaches et alimentation (nombre d'ateliers)

race

$\begin{array}{lrrrrrrr}\text { - Pie Rouge } & 5 & 12 & 10 & 6 & 3 & 11 & 0 \\ \text { - Holstein } & 1 & 1 & 3 & 4 & 2 & 0 & 3 \\ \text { - pas de race dominante } & 1 & 0 & 3 & 0 & 0 & 2 & 0\end{array}$

ration de base

- fourrage sec dominant $\quad 5 \quad 7$

- équilibre sec/fermenté ${ }^{2} \quad 1 \quad 1$

- fourrage fermenté dominant $1 \quad 5$

$\%$ de concentré dans la ration ${ }^{3}$

$\begin{array}{llllllll}<25 \% & 5 & 3 & 2 & 1 & 0 & 3 & 2 \\ 25 \text { à } 30 \% & 0 & 4 & 4 & 4 & 3 & 3 & 1 \\ >30 \% & 1 & 2 & 6 & 4 & 2 & 2 & 0 \\ \begin{array}{l}>\text { re du concentré } \\ \text { - à base de céréales } \\ \text { - riche en parois végétales }\end{array} & 5 & 4 & 8 & 4 & 1 & 4 & 3 \\ \end{array}$

Traite et hygiène

Installation de traite (nombre d'ateliers)

- pots trayeurs

- lactoducs

- salles de traite

Note d'hygiène de traite

Note de propreté de la stabulation ${ }^{4}$

$\begin{array}{lll}3 & 3 & 4 \\ 4 & 5 & 10 \\ 0 & 5 & 2 \\ 4,4 & 5,6 & 5,6 \\ 6,8 & 5,9 & 6,0\end{array}$

$\begin{array}{lllll}2 & 2 & 5 & 0 & \text { NS } \\ 4 & 2 & 8 & 0 & \\ 4 & 1 & 0 & 3 & \\ 6,8 & 4,2 & 5,5 & 4,0 & \text { NS } \\ 6,1 & 6,1 & 6,6 & 3,5 & \text { NS }\end{array}$

${ }^{1}$ signification statistique : NS : non significatif, $+: 10 \%, *: 5 \%, * *: 1 \%$. L'analyse statistique a été réalisée sur les 64 fromages des classes 1 à $6 .{ }^{2}$ fermenté = ensilage et/ou balle ronde enrubannée. ${ }^{3}$ cette variable n'a pu être élaborée que pour 52 des 67 fromages. ${ }^{4}$ plus la note est élevée, moins la stabulation est propre.

${ }^{1}$ statistical signification: NS: non significant, $+: 10 \%, *: 5 \%, * *: 1 \%$. The statistical analysis was realized on 64 cheeses from the groups 1 to $6 .{ }^{2}$ fermented= silage and/or wrapped round bale. ${ }^{3}$ it was possible to elaborate this variable only for 52 of the 67 cheeses. ${ }^{4}$ the minor the score is, the cleaner the stable is.

variabilité peut être due à différents types de facteurs allant des conditions de production du lait jusqu' aux conditions d'affinage du fromage, en passant par leurs conditions de fabrication. Dans cette étude, la durée d'affinage était voisine pour tous les fromages et le nombre d'affineurs réduit, de sorte que la variabilité des caractéristiques des fromages n'a été qu'en partie associée à l'affinage : si trois classes de fromages seulement ont été présentes chez l'affineur 2, cinq classes sur six et six classes sur six ont été représentées chez les deux autres. En ce qui concerne les conditions de fabrication des fromages, nous avons observé une grande variabilité des pratiques d'une exploitation à l'autre, pratiques qui expliquent une partie des différences observées entre fromages.

Parmi les conditions de production du lait, les plus variables ont concerné la nature de l'alimentation, et en particulier la pro- 
portion de fourrages fermentés dans la ration, et dans une moindre mesure, la proportion de concentré. Nous n'avons pas pu mettre en évidence de relation nette entre la nature des fourrages, telle que nous l'avons appréciée dans cette enquête, et les caractéristiques sensorielles du fromage. Ainsi, les deux classes les plus extrêmes en matière de texture, de goût et d'odeur (classes I et V) ont présenté à la fois des caractéristiques du lait mis en fabrication identiques, ainsi que des types de ration de base très voisins. Les conditions d'affinage ont aussi été voisines dans ces deux groupes. Seules les pratiques de transformation permettent d'opposer ces deux classes extrêmes (tableau II). À l'inverse, les fromages des classes V et VI, qui ne diffèrent que légèrement par leur texture, ont été fabriqués à partir de lait de vaches recevant des rations très différentes. Par contre, la proportion de concentré dans la ration, dans les 49 fabrications où il a été possible de l'estimer, semble plus discriminante : quel que soit le type de ration, les faibles proportions de concentré dans la ration sont significativement associées aux fromages les moins fermes, au goût le plus intense et le plus persistant, et à l'odeur la plus intense. Bien que les caractéristiques du lait mis en œuvre soient très voisines, ces faibles proportions de concentré sont aussi associées aux fromages les plus humides et les moins riches en matières grasses. Cette étude ne permet cependant pas de conclure à un effet direct du concentré. En effet, les pratiques de fabrication different sensiblement selon la proportion de concentré dans la ration : en particulier, les fromages fabriqués à partir de lait de vaches recevant peu de concentré sont aussi ceux qui ont été fabriqués dans une salle fraîche $\left(14^{\circ} \mathrm{C}\right)$, avec peu de présure et avec un mélange de ferments mésophiles et thermophiles, alors que, pour ceux fabriqués avec le lait de vaches recevant la part la plus importante de concentré, l'utilisation de ferments thermophiles est quasi systématique, la température de la salle de fabrication est élevée $\left(19^{\circ} \mathrm{C}\right)$, de même que les doses de présure.

D'un point de vue plus global cette étude montre que l'on peut opposer des fromages typés (classes I et II) à des fromages plus neutres (classe IV). Ces deux groupes sont associés à la fois à des fabrications différentes (ferments et doses de présure) et à des systèmes de conduite des animaux différents, plus traditionnels dans le premier cas, plus intensifs dans le second (part de concentré importante, vêlages plus précoces en hiver, présence de vaches de race holstein, traite bien maîtrisée). La nature de l'étude ne permet cependant pas de savoir s'il s'agit d'une association fortuite, et dans la négative, quels sont les facteurs réellement responsables de la qualité des fromages, mais suggère que des pratiques associées à une faible intensification de la conduite alimentaire des animaux peuvent conduire à des produits plus typés.

L'objectif de cette étude était de mettre en évidence une association éventuelle entre les conditions d'alimentation des animaux et les caractéristiques sensorielles du fromage saint-nectaire fermier, comme le suggèrent les nombreuses observations empiriques des fromagers [17] et les travaux récents entrepris dans différentes conditions sur ce sujet [12]. Au vu des résultats, il n'est pas possible d'affirmer que le mode de conservation des fourrages conditionne de manière systématique les caractéristiques des fromages. Par exemple, que les rations à base de foin conduisent toujours à un fromage différent de ceux fabriqués avec du lait de vache recevant une ration à base d'ensilage. Cela va dans le sens des résultats expérimentaux que nous avons récemment obtenus $[18,19]$, qui montrent que le mode de conservation des fourrages, lorsque celle-ci était bien maîtrisée, n'a qu'un effet limité sur les caractéristiques du fromage, en dehors de la couleur.

Les résultats obtenus en fonction de la proportion de concentré dans la ration, même s'ils doivent être interprétés avec pru- 
dence, suggèrent en revanche des associations entre mode de conduite alimentaire et caractéristiques des fromages. Cependant, l'origine (mélange fermier ou concentré du commerce) et la nature du concentré (à base de céréales ou contenant une proportion sensible de parois végétales) n'est pas apparue discriminante dans cette étude. La très grande diversité des formules d'aliments concentrés utilisés par les éleveurs ne permet pas de conclure sur ce sujet à partir de ces données.

En conclusion, ce travail doit être considéré comme la première phase d'un programme plus complet concernant l'étude des relations entre facteurs de production et qualité des fromages. Il est complémentaire des approches plus analytiques réalisées par ailleurs sur des fromages fabriqués en conditions contrôlées [7, 18]. Ces résultats méritent donc d'être confirmés et précisés à l'aide de dispositifs de suivis et d'observations adéquats, concentrés sur un nombre restreint d'exploitations, résultats éventuellement confirmés par des expérimentations en conditions contrôlées.

\section{REMERCIEMENTS}

Cette étude a été réalisée dans le cadre du pôle A.O.C. fromager Massif Central. Nous tenons à remercier les producteurs de saint-nectaire et les affineurs qui ont participé à cette étude, ainsi que le syndicat de défense du saint-nectaire.

\section{RÉFÉRENCES}

[1] AFNOR, Méthodologie - Guide général pour la sélection, l'entraînement et le contrôle des sujets qualifiés, NF V 09-003, 1988.

[2] AFNOR. Méthodologie - Recherche de descripteurs pour l'élaboration d'un profil sensoriel, NF V 09-021, 1990.

[3] AFNOR, Méthode de routine pour le dénombrement des coliformes par comptage des colonies à $30^{\circ} \mathrm{C}(24 \mathrm{~h})$, XPV08050, 1992

[4] AFNOR, Méthode de routine pour le dénombrement des staphylocoques à coagulase positive par comptage des colonies à $37^{\circ} \mathrm{C}$, XPV08057-2, 1994
[5] Barthélémy J., Évaluation d'une grandeur sensorielle complexe : description quantifiée, in : Évaluation sensorielle, manuel méthodologique, Technique et documentation Lavoisier, Paris, 1990, pp.145-162.

[6] Cerf O., Bergère J.L., La numération des spores de clostridium et son application au lait et aux produits laitiers. Numération des différents groupes de clostridium, Lait 48 (1978) 501-519.

[7] Coulon J.B., Verdier I,, Pradel P., Effet du mode de présentation du même fourrage (foin ou herbe pâturée) sur les caractéristiques de fromages de Saint-Nectaire affinés, Lait 76 (1996) $479-486$.

[8] Faye B., Barnouin J., Objectivation de la propreté des vaches laitières et des stabulations. L'indice de propreté, Bull. Tech. C.R.Z.V. Theix INRA 59 (1985) 61-67.

[9] FIL-IDF, Détermination de la teneur en acides gras libres, norme 265, 1991.

[10] Garel J.P., Coulon J.B., Effet de l'alimentation et de la race des vaches sur la fabrication de fromage d'Auvergne de saint-nectaire, INRA Prod. Anim. 3 (1990) 127-136.

[11] Grandison A.S., Manning D.J.. Thomson D.J., Anderson M., Interrelationships between the diet fed to cows, composition and properties of milk and composition and quality of Cheshire cheese from farmhouse manufacturers, J. Dairy Res. 52 (1985) 587-593.

[12] Grappin R., Coulon J.B., Terroir, lait et fromage : éléments de réflexion, Renc. Rech. Ruminants 3 (1996) 21-28.

[13] Martin B., Influence des pratiques d'élevage et de fabrication fromagère sur les caractéristiques du lait et du fromage. Cas du reblochon fermier, thèse de l'École nationale supérieure agronomique de Montpellier, 1993.

[14] Martin B., Coulon J.B., Facteurs de production du lait et caractéristiques des fromages. II. Influence des caractéristiques des laits de troupeaux et des pratiques fromagères sur les caractéristiques du reblochon de Savoie fermier, Lait 75 (1995) 133-149.

[15] Martin B., Coulon J.B., Chamba J.F.. Bugaud C., Effect of milk urea on characteristics of matured Reblochon cheeses, Lait 77 (1997) 505-514.

[16] SAS Institute Inc., SAS User's Guide: Statistics, SAS Institute Inc, Cary, NC, 1987.

[17] Urbach G., Effect of feed on flavour in dairy foods, J. Dairy Sci. 73 (1990) 3639-3650.

[18] Verdier I., Coulon J.B., Pradel P., Berdagué J.L., Effect of forage type and cow breed on the characteristics of matured Saint-Nectaire cheeses, Lait 75 (1995) 523-533.

[19] Verdier I., Coulon J.B., Pradel P., Viallon C., Berdagué J.L., Effect of forage conservation (hay or silage) and cow breed on the coagulation properties of milk and on the characteristics of ripened cheeses, J. Dairy Res. 65 (1998) 9-21. 